\title{
Lemniscate Growth
}

\section{Erik Lundberg • Vilmos Totik}

Received: date / Accepted: date

Abstract It was recently noticed that lemniscates do not survive Laplacian growth [12] (2010). This raises the question: "Is there a growth process for which polynomial lemniscates are solutions?" The answer is "yes", and the law governing the boundary velocity is reciprocal to that of Laplacian growth.

Viewing lemniscates as solutions to a moving-boundary problem gives a new perspective on results from classical potential theory, and interesting properties emerge while comparing lemniscate growth to Laplacian growth.

Keywords lemniscate $\cdot$ Laplacian growth $\cdot$ elliptic growth $\cdot$ commuting flows · electrostatic skeleton

The second author acknowledges support from the European Research Council grant No. 267055 .

E. Lundberg

Department of Mathematics

Purdue University

150 N. University Street, West Lafayette, IN 47907-2067

E-mail: elundber@math.purdue.edu

V. Totik

Bolyai Institute

Analysis and Stochastics Research Group of the Hungarian Academy of Sciences

University of Szeged, Szeged

Aradi v. tere 1, 6720, Hungary

and

Department of Mathematics and Statistics

University of South Florida

4202 E. Fowler Ave, PHY 114

Tampa, FL 33620-5700, USA

E-mail: totik@mail.usf.edu 


\section{Introduction: Lemniscate growth is "reciprocal" to Laplacian growth}

In this work we heavily rely on concepts (like Green's functions, harmonic measure, equilibrium measure or balayage) and basic result from potential theory. For these see [19], [14], [23] or [22].

Suppose $\left\{\mathcal{L}_{t}\right\}$ is a one-parameter family of domains in $\mathbb{C} \cong \mathbb{R}^{2}$, and let $L_{t}$ be the boundary curves. Recall that $\left\{\mathcal{L}_{t}\right\}$ is called a Laplacian growth process with sink at $z_{0} \in \mathcal{L}_{t}$ if the normal component of the boundary velocity $V(z)$ at a point $z \in L_{t}$ coincides with the normal derivative of Green's function with pole at $z_{0} \in \mathcal{L}_{t}$ :

$$
V(z)=\partial_{n} g_{\mathcal{L}_{t}}\left(z, z_{0}\right) .
$$

We are especially interested in the case when the domains $\left\{\mathcal{L}_{t}\right\}$ are unbounded and $z_{0}=\infty$. We note that we are taking the convention that $g_{\mathcal{L}_{t}}\left(z, z_{0}\right)$ has a positive singularity at $z_{0}$, so that (1) defines a shrinking domain.

It was observed in [12] that lemniscates are not preserved under Laplacian growth, except in the trivial case of a circle. Namely, the authors proved the following

Theorem. Suppose that a family of moving boundaries $L_{t}$, (where $t>0$ is time), produced by a Laplacian growth process, is a family of polynomial lemniscates $|P(z, t)|=1$, where $P(z, t)=a(t) \prod_{j=1}^{n}\left[z-\lambda_{j}(t)\right]$, and all $\lambda_{j}(t)$ are assumed to be inside $L_{t}$. Then, $L_{t}$ is a family of concentric circles.

This raises an obvious question.

Question: Is there a growth process that preserves lemniscates?

The answer is "yes" and it is precisely the reciprocal of the Laplacian growth law described by Eq. (1). To be explicit, consider the problem of finding a one-parameter family of domains $\Omega_{t}$ so that the normal component $V(z)$ of the velocity of the boundary satisfies

$$
V(z)=\frac{1}{\partial_{n} g_{\Omega_{t}}\left(z, z_{0}\right)} .
$$

Then an evolution of polynomial lemniscates (different level sets of the modulus of a single polynomial) gives an exact solution to this problem with $z_{0}=\infty$.

Example 1 Let $T_{N}$ be a fixed polynomial of degree $N$, and consider the oneparameter family of domains $\Sigma_{t}=\left\{z|| T_{N}(z) \mid>\exp \{N t\}\right\}$. Then the normal velocity $V(z)$ of the boundary satisfies (2) with $z_{0}=\infty$ and with $\Omega_{t}=\Sigma_{t}$.

Indeed, the lemniscate $\Gamma_{t}=\left\{z|| T_{N}(z) \mid=\exp \{N t\}\right\}$ is the boundary of $\Sigma_{t}$. The Green's function $g_{\Sigma_{t}}(\cdot, \infty)$ with pole at infinity of the domain $\Sigma_{t}$ is

$$
g_{\Sigma_{t}}(z, \infty)=\frac{1}{N} \log \left|T_{N}(z)\right|-t
$$

Notice that $\Gamma_{t}$ is the zero set of $g_{\Sigma_{t}}(z, \infty)$, but it is also the $t$-set of $g_{\Sigma_{0}}(z, \infty)$, i.e., $\Gamma_{t}=\left\{z\left|\frac{1}{N} \log \right| T_{N}(z) \mid=t\right\}$. 
We recall a general principle. If $\Gamma_{t}:=\{z \mid F(z)=t\}$ are the level sets of a function $F(z)$, then taking some point $z$ on $\Gamma_{t}$ and moving in the normal direction by $\Delta z$ to obtain a point on some other level curve $\Gamma_{t+\Delta t}$, we have

$$
\frac{F(z+\Delta z)-F(z)}{|\Delta z|}=\frac{\Delta t}{|\Delta z|} .
$$

Thus, $\partial_{n} F(z)$ is the reciprocal of the velocity of the level curves. In our case, $F(z)=\frac{1}{N} \log \left|T_{N}(z)\right|$, and we have

$$
V(z)=\frac{1}{\partial_{n} g_{\Sigma_{0}}(z, \infty)} .
$$

But since $g_{\Sigma_{t}}(z, \infty)=g_{\Sigma_{0}}(z, \infty)-t$, then $\partial_{n} g_{\Sigma_{0}}(z, \infty)=\partial_{n} g_{\Sigma_{t}}(z, \infty)$ and $(2)$ holds for $\Omega_{t}=\Sigma_{t}$.

We propose to call any such process governed by (2) "lemniscate growth" based on the many exact solutions provided by lemniscates. The purpose of this survey is first to revisit some results from classical potential theory in the setting of lemniscate growth, second, to make comparisons to Laplacian growth, and third, to draw connections to some studies in disparate areas: domain reconstruction [8], (mem)Brane theory [3], elliptic growth [11], and non-Newtonian Hele-Shaw flows [5].

In Section 2, we explore basic properties following directly from classical potential theory. In Section 3, we discuss a conservation law resembling Richardson's theorem. In Section 4, we include a strength parameter $\nu(t)$ in (2):

$$
V(z)=\frac{\nu(t)}{\partial_{n} g_{\Omega_{t}}\left(z, z_{0}\right)},
$$

and consider the reverse process, when $\nu$ is negative, so that the domain $\Omega_{t}$ is growing. The well-posedness is more delicate in this case and large time existence relates to a potential theoretic question about so-called electrostatic skeletons, see Section 4. In Section 5, instead of a single Green's function we take a superposition so that there are multiple fixed singularities. An appealing aspect of Laplacian growth, recently connected to the Whitham hierarchy in integrable systems [13], is that multiple singularities give rise to "commuting flows". We observe that this property fails for lemniscate growth, but holds for a modified version of it. See the "zero-curvature" condition in Section 5 described by Equation (17). In Section 6, as an addendum to the result from [12] stated above, we work out an example showing that certain cases of elliptic growth (a generalization of Laplacian growth) admit lemniscate solutions. 


\section{Existence, uniqueness, and regularity}

Let us take any simply connected domain $\Omega_{0}$ that is not all of $\mathbb{C}$, and consider the problem of finding $\Omega_{t}$ so that (2) is satisfied. We will see that existence, uniqueness, and regularity follow from considerations in classical potential theory. The problem is not only well-posed, but also stable (approaching a circle in the limit). The reverse-process, i.e. backward-time lemniscate growth, is generally ill-posed and unstable. As with Laplacian growth, the unstable direction is perhaps more interesting, and will be discussed in Section 4 .

\subsection{Existence:}

Let $z_{0} \in \Omega_{0}$ be a fixed point. By the Riemann mapping theorem, there is a conformal map $f: \Omega_{0} \rightarrow \mathbb{C} \backslash \overline{\mathbb{D}}$, with $f\left(z_{0}\right)=\infty$. The Green's function $g_{\Omega_{0}}\left(z, z_{0}\right)$ of $\Omega_{0}$ with pole at $z_{0}$ equals $\log |f(z)|$. Let $\Omega_{t}=\{z|\log | f(z) \mid>t\}$. Then the Green's function $g_{\Omega_{t}}\left(z, z_{0}\right)$ is $\log |f(z)|-t$, and we can argue exactly as in Example 1 that the boundary of $\Omega_{t}$ moves with velocity given by (2).

This proves existence, but let us give some additional detail. The curves orthogonal to $\Gamma_{t}:=\partial \Omega_{t}$ are trajectories of an ordinary differential equation. In what follows we write $z=x+i y$ and $\partial u / \partial z=\left(u_{x},-u_{y}\right)=u_{x}-i u_{y}$. Let $g(z)=g_{\Omega_{0}}\left(z, z_{0}\right)$. Consider the vector field

$$
F(z)=\frac{\nabla g}{|\nabla g|^{2}}=\left(\frac{g_{x}}{g_{x}^{2}+g_{y}^{2}}, \frac{g_{y}}{g_{x}^{2}+g_{y}^{2}}\right)=\frac{1}{\partial g / \partial z} .
$$

This is analytic since $\partial g / \partial z$ is analytic. Consider the trajectory $\gamma_{s}(t)$ of a point $s \in \Gamma_{0}$ in this vector field. This means that the point moves at any $z$ with velocity $F(z)$, i.e.

$$
\gamma_{s}(t)^{\prime}=F\left(\gamma_{s}(t)\right), \quad t>0 ; \quad \gamma_{s}(0)=s,
$$

where $(\cdot)^{\prime}$ indicates differentiation with respect to $t . \partial g / \partial z$ is analytic in $\Omega_{0}$, and its antiderivative $H(z)$ is a (multivalued) analytic function with real part equal to $g$. Now (5) gives

$$
H^{\prime}\left(\gamma_{s}(t)\right) \gamma_{s}(t)^{\prime}=1
$$

so by integration we get that (taking an appropriate branch of $H) H\left(\gamma_{s}(t)\right)=$ $t+c$, where the constant $c$ is imaginary (since $\operatorname{Re}\left(H\left(\gamma_{s}(0)\right)=g\left(\gamma_{s}(0)\right)=0\right)$. Thus, $g\left(\gamma_{s}(t)\right)=t$, i.e. the points $\gamma_{s}(t), s \in \Gamma_{0}$, lie on the level curve $\{z \mid g(z)=$ $t\}$. The curves $\gamma_{s}(t), t>0$, are called Green lines, and they are orthogonal to the boundary curves $\Gamma_{t}$ (this follows from the fact that $\gamma_{s}(t)$ moves in the direction of the gradient of $g$ which is orthogonal to the level curves $\Gamma_{t}$ ). These Green lines run from $\Gamma_{0}$ to $z_{0}$. 
Example 2 Consider the potential field

$$
G(z)=\int \log |z-\xi| d \mu(\xi)
$$

generated by a unit charge distribution $\mu$ lying exterior to $\Omega_{0}:=\{z \mid G(z)>$ $\left.v_{0}\right\}$. Then $z_{0}=\infty, g(z)=G(z)-v_{0}$, and the Green lines are the paths of charged particles when placed in this potential field.

If the total charge of $\mu$ is not 1 but $1 / \lambda$, then $g(z)=\lambda\left(G(z)-v_{0}\right)$, and in this case the level sets $\{z \mid G(z)=t\}$ move with velocity equal to $\lambda$ times the reciprocal of the normal derivative.

\subsection{Uniqueness:}

Suppose $\left\{\tilde{\Omega}_{t}\right\}$ is another growth process where the boundary moves with velocity equal to the reciprocal of the normal derivative of the Green's function $g_{\tilde{\Omega}_{t}}\left(\cdot, z_{0}\right)$ and $\tilde{\Omega}_{0}=\Omega_{0}$. To show that $\tilde{\Omega}_{t}=\Omega_{t}$, consider the level domains of the Green's function $g_{\tilde{\Omega}_{t}}\left(\cdot, z_{0}\right)$ :

$$
\tilde{\Omega}_{t, \tau}=\left\{z \mid g_{\tilde{\Omega}_{t}}\left(z, z_{0}\right)>\tau\right\}, \quad \tau>0 .
$$

For this

$$
g_{\tilde{\Omega}_{t, \tau}}\left(\cdot, z_{0}\right)=g_{\tilde{\Omega}_{t}}\left(\cdot, z_{0}\right)-\tau
$$

Since, in small time $\tau>0$ a boundary point $z \in \tilde{\Gamma}_{t}$ moves to a point $z(\tau)$ where the value $g_{\tilde{\Omega}_{t}}\left(z(\tau), z_{0}\right)$ is about $\tau$, we get that no matter how small $\eta>0$ is, for small $\tau>0$ we have

$$
\tilde{\Omega}_{t,(1+\eta) \tau} \subset \tilde{\Omega}_{t+\tau} \subset \tilde{\Omega}_{t,(1-\eta) \tau},
$$

so at a point $w \in \tilde{\Omega}_{t}$ we have for small $\tau$ the inequality

$$
g_{\tilde{\Omega}_{t}}\left(w, z_{0}\right)-(1-\eta) \tau \geq g_{\tilde{\Omega}_{t+\tau}}\left(w, z_{0}\right) \geq g_{\tilde{\Omega}_{t}}\left(\cdot, z_{0}\right)-(1+\eta) \tau .
$$

Thus,

$$
\frac{\partial g_{\tilde{\Omega}_{t}}\left(w, z_{0}\right)}{\partial t}=-1
$$

and so

$$
g_{\tilde{\Omega}_{t}}\left(w, z_{0}\right)=g_{\tilde{\Omega}_{0}}\left(w, z_{0}\right)-t .
$$

But the right-hand side is just $g_{\Omega_{t}}\left(w, z_{0}\right)$, so

$$
g_{\tilde{\Omega}_{t}}\left(w, z_{0}\right)=g_{\Omega_{t}}\left(w, z_{0}\right),
$$

which shows (say upon letting $w$ tending to the boundary of $\tilde{\Omega}_{t}$ ) that $\tilde{\Omega}_{t}=\Omega_{t}$ for all $t$. 
2.3 Regularity:

Since the boundaries $\partial \Omega_{t}$ are images of circles under a conformal map, they foliate $\Omega_{0}$ by analytic, non-singular curves shrinking to the point $z_{0}$.

\section{Conformal invariance and conserved quantities}

Since Green's functions are conformally invariant, it follows from the above discussion that so is lemniscate growth: if $\varphi$ is a conformal map of $\Omega_{0}$ onto $\tilde{\Omega}_{0}$, then the process $\left\{\tilde{\Omega}_{t}\right\}$ starting at $\varphi\left(\Omega_{0}\right)$ with pole at $\varphi\left(z_{0}\right)$ is the same as the image of the process $\left\{\Omega_{t}\right\}$ starting at $\Omega_{0}$ with pole at $z_{0}: \varphi\left(\Omega_{t}\right)=\tilde{\Omega}_{t}$.

Let $\sigma_{t}=\sigma_{t, z_{0}}$ be the harmonic measure of the point $z_{0}$ with respect to the domain $\Omega_{t}$. This means that $\sigma_{t}$ is a unit measure on $\Gamma_{t}$ such that for any function $u$ which is harmonic in $\Omega_{t}$ and continuous on the closure of $\Omega_{t}$ we have

$$
u\left(z_{0}\right)=\int_{\Gamma_{t}} u d \sigma_{t} .
$$

Thus, the integrals of functions harmonic in $\Omega_{0}$ against the measures $\sigma_{t}$ are conserved during the process.

It is known (cf. [22, (I.4.8) and Theorem II.1.5]) that if $d s_{t}$ is the arc element of $\Gamma_{t}$, then

$$
d \sigma_{t, z_{0}}=\frac{1}{2 \pi} \frac{\partial g\left(\cdot, z_{0}\right)}{\partial \mathbf{n}} d s_{t} .
$$

The preceding formula shows that for $t_{1}<t_{2}$ the measure $\sigma_{t_{2}}$ is the balayage of the measure $\sigma_{t_{1}}$ onto $\Gamma_{t_{2}}$ (out of $\overline{\mathbb{C}} \backslash \overline{\Omega_{t_{2}}}$ ). The reverse, i.e. that for $t_{1}<t_{2}$ the measure $\sigma_{t_{1}}$ is the balayage of the measure $\sigma_{t_{2}}$ onto $\Gamma_{t_{1}}$ (out of $\Omega_{t_{1}}$ ) is also true, and follows from the next invariance. Also note that in the case $z_{0}=\infty$ the measure $\sigma_{t}$ is precisely the equilibrium measure of $\Gamma_{t}$.

If $u$ is harmonic in $\overline{\mathbb{C}} \backslash \overline{\Omega_{t_{0}}}$, then the integrals $\int_{\Gamma_{t}} u d \sigma_{t}$ are still preserved for $0<t<t_{0}$. Indeed, take two such $t: 0<t_{1}<t_{2}<t_{0}$, and write up Green's formula for the ring domain enclosed by $\Gamma_{t_{1}}$ and $\Gamma_{t_{2}}$. We get

$$
\int_{\Gamma_{t_{1}}} u \frac{\partial g}{\partial \mathbf{n}} d s_{t_{1}}-\int_{\Gamma_{t_{2}}} u \frac{\partial g}{\partial \mathbf{n}} d s_{t_{2}}=\int_{\Gamma_{t_{1}}} g \frac{\partial u}{\partial \mathbf{n}} d s_{t_{1}}-\int_{\Gamma_{t_{2}}} g \frac{\partial u}{\partial \mathbf{n}} d s_{t_{2}} .
$$

In both integrals on the right $g$ is constant $\left(=t_{1}\right.$ resp. $\left.t_{2}\right)$, and, again by Green's formula,

$$
\int_{\Gamma_{t_{j}}} \frac{\partial u}{\partial \mathbf{n}} d s_{t_{j}}=0, \quad j=1,2 .
$$

So the left-hand side is zero for all $t_{1}$ and $t_{2}$, and we obtain that

$$
\int_{\Gamma_{t}} u d \sigma_{t}
$$


is constant. In the case of Example 2 we get

$$
\int_{\Gamma_{t}} u d \sigma_{t}=\lambda \int u d \mu
$$

and in the case of lemniscates $\Gamma_{t}=\left\{z|| T_{N}(z) \mid=t\right\}$ we have

$$
\int_{\Gamma_{t}} u d \sigma_{t}=\frac{1}{|Z|} \sum_{z \in Z} u(z)
$$

where the summation is taken for the zero set $Z$ of $T_{N}$ (counting multiplicities).

This formula can be summarized by saying that lemniscates are a type of generalized quadrature domain, namely, they admit a quadrature formula for integration of harmonic functions w.r.t. equilibrium measure.

When $z_{0}=\infty$, then the convexity of the boundary curves $\Gamma_{t}$ is preserved. Indeed, let $\varphi=\varphi_{\Omega_{0}}$ be the conformal map from the exterior of the unit disk onto $\Omega_{0}$. Then $g_{\Omega_{0}}=\log \left|\varphi^{-1}\right|$, and $\Gamma_{t}=\varphi\left(C_{t}\right)$, where $C_{t}$ is the circle $\{z|| z \mid=t\}$. The convexity of $\Gamma_{0}$ is equivalent to the condition (see [18, Theorem 2.9])

$$
\operatorname{Re} z \frac{\varphi^{\prime}(z)}{\varphi(z)}>0, \quad|z|>1
$$

Now $\varphi_{\Omega_{t}}(z)=\varphi_{\Omega_{0}}(t z)$, and then $(9)$ implies

$$
\operatorname{Re} z \frac{\varphi_{\Omega_{t}}^{\prime}(z)}{\varphi_{\Omega_{t}}(z)}=z t \frac{\varphi_{\Omega_{0}}^{\prime}(z t)}{\varphi_{\Omega_{0}}(z t)}>0, \quad|z|>1 .
$$

\subsection{A minimality property}

The curves $\Gamma_{t}, 0<t<T$, are pairwise disjoint and they fill the region $\mathcal{G}_{T}$ lying in between $\Gamma_{0}$ and $\Gamma_{T}$. The same is true of the orthogonal family of Green lines $\gamma_{s}, s \in \Gamma_{0}$. Now there are many other smooth families $\tilde{\Gamma}_{t}$ connecting $\Gamma_{0}$ and $\Gamma_{T}$, and for them we can form the corresponding orthogonal family $\tilde{\gamma}_{s}(t)$ parametrized so that $\tilde{\gamma}_{s}(t) \in \tilde{\Gamma}_{t}, \tilde{\gamma}_{s}(0)=s \in \Gamma_{0}$. Let $s=s(\tau), \tau \in[0,2 \pi]$, be a parametrization of $\Gamma_{0}$. Then $\tilde{\gamma}(\tau, t):=\tilde{\gamma}_{s(\tau)}(t), \tau \in[0,2 \pi], 0<t<T$, is a parametrization of the points in the domain $\mathcal{G}_{T}$. Using the argument at the beginning we get then that if the function $u$ is defined on $\mathcal{G}_{T}$ by $u(\tilde{\gamma}(\tau, t))=t$, then $\tilde{\Gamma}_{t}$ are the level curves of $u$ and

$$
\tilde{\gamma}_{t}=\frac{\partial \tilde{\gamma}}{\partial t}=\frac{\nabla u}{|\nabla u|^{2}}=\left(\frac{u_{x}}{u_{x}^{2}+u_{y}^{2}}, \frac{u_{y}}{u_{x}^{2}+u_{y}^{2}}\right) .
$$

Now

$$
\frac{1}{2 \pi} \int_{\tilde{\Gamma}_{t}} \frac{1}{\left|\tilde{\gamma}_{t}\right|} d \tilde{s}_{t}(\tau)=\frac{1}{2 \pi} \int_{0}^{2 \pi} \frac{\left|\tilde{\gamma}_{\tau}\right|}{\left|\tilde{\gamma}_{t}\right|} d \tau
$$


is the average reciprocal speed of the boundary on $\tilde{\Gamma}_{t}$ (sort of average resistance when transferring charges, since $1 /\left|\tilde{\gamma}_{t}\right|$ is the time needed to transfer a fixed but small amount of charge), and

$$
\tilde{V}:=\frac{1}{2 \pi T} \int_{0}^{T} \int_{\tilde{\Gamma}_{t}} \frac{1}{\left|\tilde{\gamma}_{t}\right|} d \tilde{s}_{t}(\tau) d t=\frac{1}{2 \pi T} \int_{0}^{T} \int_{0}^{2 \pi} \frac{\left|\tilde{\gamma}_{\tau}\right|}{\left|\tilde{\gamma}_{t}\right|} d \tau d t
$$

can be called the average reciprocal speed of the boundary of the motion $\left\{\Gamma_{t}\right\}$. We always have $\tilde{V} \geq 1$ with equality only for the lemniscate growth. Indeed, $\left|\tilde{\gamma}_{\tau}\right|\left|\tilde{\gamma}_{t}\right| d \tau d t$ is the area element, and $\left|\tilde{\gamma}_{t}\right|^{-2}=|\nabla u|^{2}$. Hence $\tilde{V}$ is nothing else than the Dirichlet integral

$$
\frac{1}{2 \pi T} \int_{\mathcal{G}_{T}}|\nabla u|^{2},
$$

and on the boundary of $\mathcal{G}_{T}$ the function $u$ coincides with $g$. By Dirichlet's principle ([23, Section I.5]) this integral is minimal when $u$ is harmonic, i.e. when it agrees with $g$. In this case $\tilde{\gamma}=\gamma$, and on $\Gamma_{t}$ we have (see (7))

$$
\frac{1}{2 \pi} \frac{\left|\gamma_{\tau}\right|}{\left|\gamma_{t}\right|} d \tau=\frac{1}{2 \pi} \frac{1}{\left|\gamma_{t}\right|} d s_{t}=\frac{1}{2 \pi} \frac{\partial g}{\partial \mathbf{n}} d s_{t}=d \sigma_{t}
$$

so all the inner integrals in (10) are equal to $2 \pi$, from which $\tilde{V}=1$ follows in this case.

\section{The reverse process, electrostatic skeletons}

In general, if $\lambda$ is a parameter and the boundary of $\Omega_{t}$ moves with velocity equal to $\lambda$ times the reciprocal of the normal derivative, then the only difference in the above construction is that, instead of (5), we have

$$
\gamma_{s}(t)^{\prime}=\lambda F\left(\gamma_{s}(t)\right), \quad t>0 ; \quad \gamma_{s}(0)=s,
$$

the solution of which is $g\left(\gamma_{s}(t)\right)=\lambda t$, so it is the same process, just speeded up. In a similar manner, if $\lambda$ is a negative parameter, then we still get the same process, but going backward in time (i.e. in this case the domains $\Omega_{t}$ are growing, while in the $\lambda>0$ case they are shrinking).

For definiteness consider the case when the pole $z_{0}$ is at infinity. Starting from a nice domain $\Omega_{0}$ (containing $\infty$ ) with an analytic Jordan curve as its boundary, the process can run backwards so long as the boundary consists of a single analytic curve. Further continuation may be possible by allowing non-simply connected domains.

Let $t_{0}$ be the smallest number for which the process can run backward for all $t>t_{0}$.

Suppose that, as in an ideal case, there is a compact set $K$ of positive capacity with connected complement and empty interior, and $\cup_{t>t_{0}} \Omega_{t}=\overline{\mathbb{C}} \backslash$ $K$. Then, in $\Omega_{0}$, the equilibirum measure of the set $K$ generates the same logarithmic potential as the equilibrium measure of $\mathbb{C} \backslash \overline{\Omega_{0}}$ (which in turn is 
the same as the equilibrium measures of $\left.\Gamma_{0}=\partial \Omega_{0}\right)$. This follows from the fact that for $z_{0}=\infty$ the measure $\sigma_{t}$ in (6) is the equilibrium measure of $\Gamma_{t}$ and, as $t \rightarrow t_{0}$, the equilibrium measures of $\Gamma_{t}=\partial \Omega_{t}$ tend to the equilibrium measure of $K$.

More generally, a measure $\mu$ supported on (but not necessarily the equilibrium measure of) a compact $K \subset \overline{\mathbb{C}} \backslash \Omega_{0}$ with empty interior and connected complement is called an electrostatic skeleton of $\Omega_{0}$ if it generates the same logarithmic potential as the equilibrium measure of $\mathbb{C} \backslash \Omega_{0}$.

The problem if an electrostatic skeleton exists is essentially an inverse balayage problem. Indeed, if $\mu$ is supported on $K$ and in $\Omega_{0}$ we have

$$
\int \log |z-t| d \mu(t)=\int \log |z-t| d \sigma_{0}(t), \quad z \in \Omega_{0},
$$

where $\sigma_{0}$ is the equilibrium measure of $\Gamma_{0}$, then the balayage of $\mu$ onto $\Gamma_{0}$ (out of $\left.\overline{\mathbb{C}} \backslash \overline{\Omega_{0}}\right)$ must coincide with $\sigma_{0}$. The converse is also true: if the balayage of $\mu$ onto $\Gamma_{0}$ coincides with $\sigma_{0}$, then (11) holds. Thus, the problem of electrostatic skeleton is to find an appropriate set $K \subset \overline{\mathbb{C}} \backslash \Omega_{0}$ and a measure $\mu$ on $K$ such that the equilibrium measure of the boundary $\Gamma_{0}$ is the balayage of $\mu$ onto $\Gamma_{0}$.

Example 3 Let $K$ be a compact subset of $\mathbb{C}$ with connected complement and empty interior, and let $\mu$ be a unit measure on $K$. Set

$$
M=\max \int \log |z-t| d \mu(t)
$$

and for a $v_{0}>M$ consider

$$
\Omega_{0}=\left\{z\left|\int \log \right| z-\xi \mid d \mu(\xi)>v_{0}\right\} .
$$

Then $\mu$ is an electrostatic skeleton of $\Omega_{0}$, and the growth process

$$
\Omega_{t}=\left\{z\left|\int \log \right| z-\xi \mid d \mu_{K}(\xi)>v_{0}+t\right\}, \quad t \geq 0,
$$

can run backward to at least the time point $M-v_{0}$.

Consider, for instance, a polynomial lemniscate $\Omega_{0}=\left\{z|| T_{N}(z) \mid=1\right\}$ with $N$ poles as in the introduction. This corresponds to the case of Example 3 when $K$ is the zero set of $T_{N}$, and $\mu$ is the normalized counting measure on the zeros. In this case $M=-\infty$, so the process can run backward forever. During the reverse process the boundary curve breaks into $N$ components, and in the long run these components shrink into single points.

Example 4 Let now $K$ be a regular compact subset of $\mathbb{C}$ (regular with respect to the Dirichlet problem in $\overline{\mathbb{C}} \backslash K$ ) with connected complement and empty interior, and let $\mu$ be the equilibrium measure of $K$. In that case

$$
M=\max \int \log |z-t| d \mu(t)=\log \operatorname{cap}(K),
$$


where $\operatorname{cap}(K)$ denotes the logarithmic capacity of $K$, and the process in Example 3 can run backward precisely to time $t_{0}=\log \operatorname{cap}(K)-v_{0}$, and the domains $\Omega_{t}, t>t_{0}$ cover the whole complement of $K$.

A special case of this is when $\Omega_{0}$ is the complement of an ellipse: in that case $\Omega_{0}$ has an electrostatic skeleton which is the equilibrium measure of the focal segment.

Let us refer to $\mu$ as an equilibrium skeleton in the special case when it is the equilibrium measure of its support, as in Example 4 above. Actually, Example 4 describes all equilibrium skeletons with positive capacity for which the domains $\Omega_{t}$ fill the complement of $K$ : if $K$ is of positive capacity, then

$$
g_{\Omega_{t}}(z, \infty)=g_{\overline{\mathbb{C}} \backslash K}(z, \infty)+t_{0}-t, \quad t>t_{0}, z \in \Omega_{t} .
$$

Example 5 Let $\Omega_{0}$ be the infinite complement of a regular $n$-gon, and $\Gamma_{0}=$ $\partial \Omega_{0}$. We show that in this case $\Omega_{0}$ has an electrostatic skeleton which is not an equilibrium skeleton.

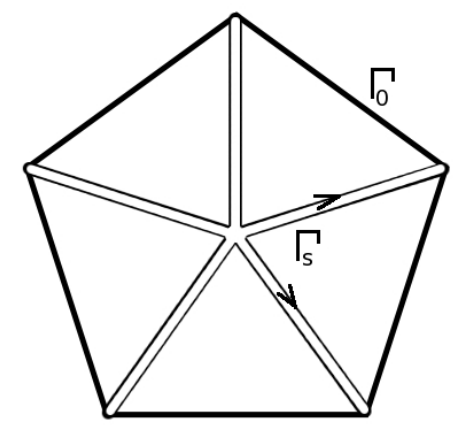

Let $S$ be the star shaped union of the segments joining the center of $\Omega_{0}$ to each vertex, and let $\Gamma_{S}$ be the boundary of the domain $\overline{\mathbb{C}} \backslash S$. Note that, as we circle around $\Gamma_{S}$, we traverse each segment of $S$ twice in opposite directions, see Figure 4.

Let $g$ be the Green's function of $\Omega_{0}$ with pole at infinity. Since $g$ is a harmonic function vanishing on $\Gamma_{0}=\partial \Omega_{0}$, the reflection principle extends $g$ across each segment by odd reflection. Let $w \in \Omega_{0}$ and consider the function $u(z)=\log |w-z|$. If $\Gamma_{t}, t>0$, is the boundary of the domain $\Omega_{t}$ obtained from $\Omega_{0}$ by lemniscate growth (with pole at infinity), then, for small $t, u$ is harmonic in the ring domain lying in between $\Gamma_{S}$ and $\Gamma_{t}$. By Green's formula for this ring domain,

$$
\int_{\Gamma_{t}} u \frac{\partial g}{\partial \mathbf{n}} d s-\int_{\Gamma_{S}} u \frac{\partial g}{\partial \mathbf{n}} d s=\int_{\Gamma_{t}} g \frac{\partial u}{\partial \mathbf{n}} d s-\int_{\Gamma_{S}} g \frac{\partial u}{\partial \mathbf{n}} d s
$$


from which we get for $t \rightarrow 0$

$$
\int_{\Gamma_{0}} u \frac{\partial g}{\partial \mathbf{n}} d s-\int_{\Gamma_{S}} u \frac{\partial g}{\partial \mathbf{n}} d s=\int_{\Gamma_{0}} g \frac{\partial u}{\partial \mathbf{n}} d s-\int_{\Gamma_{S}} g \frac{\partial u}{\partial \mathbf{n}} d s
$$

In the first integral on the right $g$ vanishes. In the second integral, each segment of $S$ is traced twice in opposite directions. By the symmetry of the domain $\Omega_{0}$, and since $g$ was extended by odd reflection, the value of $g$ on the two sides of any segment of $S$ is the same. But the normal derivatives $\frac{\partial u}{\partial \mathbf{n}}$ on the two sides are each other's negative, so the second integral on the right-hand side is also zero. Therefore, both integrals on the right vanish, and we have

$$
\int_{\Gamma_{0}} \log |w-\zeta| \frac{\partial g(\zeta)}{\partial \mathbf{n}} d s(\zeta)=\int_{\Gamma_{S}} \log |w-\zeta| \frac{\partial g(\zeta)}{\partial \mathbf{n}} d s(\zeta), \quad w \in \Omega_{0}
$$

In view of $(7)\left(\right.$ with $z_{0}=\infty$ ) this proves that the measure

$$
\frac{1}{\pi} \frac{\partial g(\zeta)}{\partial \mathbf{n}} d s(\zeta)
$$

defined on $S$ is an electrostatic skeleton of $\Omega_{0}$ with support $K=S$ (here $\mathbf{n}$ is either normal to $S$, and we wrote $1 / \pi$ and not $1 / 2 \pi$ as in (7) because the two sides of every segment of $S$ must be combined together).

The above example was worked out (but not published) by E. Miña-Diaz and E. B. Saff [21]. Some examples of irregular polygons have also been discussed by Miña-Diaz, Saff, and N. Stylianopoulos [17].

It is an open problem, due to E. B. Saff [21], which sets have an electrostatic skeleton. In particular, does the (infinite) complement of any convex polygon have an electrostatic skeleton? In other words, if $\Gamma$ is a convex polygon, is there a $\mu$ with support $K$ (having empty interior and connected complement) lying inside $\Gamma$ such that outside $\Gamma$ the measure $\mu$ and the equilibrium measure of $\Gamma$ generate the same (logarithmic) potential field?

This problem is seemingly analogous to the skeleton problem solved in [9] for area measures (which is to generate the same potential that the area measure generates), but actually it is very different: while the latter one is additive in many cases (the skeleton of a union is the union of skeletons), the former one is never additive. As explained in [9], the "mother body" (inverse balayage of area measure) for a convex polygon is supported on some union of segments lying on the angle bisectors. For the regular n-gon (Example 5 above) the electrostatic skeleton (or "Madonna body", cf. [8]) happens to have support located on the angle bisectors, but for irregular polygons this need not be the case, and the support typically includes nontrivial curves [17]. 
4.1 The multiply connected case

If $\Omega$ is $m$-connected, then the Green's function $g_{\Omega_{0}}$ may have at most $(m-1)$ critical points where the vector field $F$ in (4) is not analytic (has poles), and some of the Green lines may terminate at critical points. Still, the process $\Omega_{t}$ is continuous and the boundary curves $\Gamma_{t}$ are analytic with finitely many exceptions of the parameter $t$ where the domain changes connectivity reminiscent of bubbles merging in Laplacian growth (see the example in Section 6 below). Conformal invariance, and the invariance of integrals against harmonic measures still hold. For large $t$ the domain $\Omega_{t}$ becomes simply connected.

\section{Multiple singularities and commuting flows}

Following [24] and others, we consider the Laplacian growth process with multiple singularities by taking a superposition of Green's functions in (1):

$$
V(z)=\sum_{i=1}^{m} \nu_{i} \partial_{n} g_{\mathcal{L}_{t}}\left(z, z_{i}\right),
$$

where $z_{i}$ are in $\mathcal{L}_{t}$. Similarly, modify the condition (2) for lemniscate growth:

$$
V(z)=\frac{1}{\sum_{i=1}^{m} \nu_{i} \partial_{n} g_{\Omega_{t}}\left(z, z_{i}\right)},
$$

An interesting property of lemniscate growth emerges: the interior and exterior domains of a single evolution of polynomial lemniscates $\left\{z|| T_{N}(z) \mid>\right.$ $\left.e^{N t}\right\}$ simultaneously solve separate problems. Namely, the exterior of the curve $\Gamma_{t}$ shrinks according to (2) with singularity at $z_{0}=\infty$ (by Example 1), while the interior of $\Gamma_{t}$ grows according to (13) if we take the points $z_{i}$ to be at the zeros of the polynomial $T_{N}(z)$ and $\nu_{i}=1 / N$. Indeed, notice that the balayage measure of $\delta_{z_{i}}$ out of the interior domain $\Omega_{t}$ is $\left(\partial_{n} g_{\Omega_{t}}\left(z, z_{i}\right) / 2 \pi\right) d s(z)$, so the total balayage is their sum. If we divide this by $N$, then the so obtained measure has constant potential on the boundary, so it is the equilibrium measure, and we can use (7).

Except for concentric circles, this is never the case for quadrature domain solutions of Laplacian growth. Only one side of the curve (interior or exterior) can be a Laplacian growth. To give some idea, suppose that a quadrature domain has a Schwarz function that extends meromorphically into the domain with only simple poles and real residues. Then an evolution of such a quadrature domain with poles at fixed positions solves the problem with the weights $\nu_{i}$ determined by the rates of change of the residues. The exterior domain cannot be interpreted as a Laplacian growth, because the Schwarz function has time-dependent branch cut singularities (except in the case of a circle).

Intimately connected with Richardson's theorem [20] is the fact that Laplacian growth involving multiple singularities gives rise to commuting flows. For instance, consider two separate sinks, positioned at $a$ and $b$, and some initial 
domain $\Omega_{0}$. Imagine evolving the domain for some amount of time $T^{(a)}$ according to (1) using the normal derivative of the Green's function $g(z, a)$. Then run the process for some additional amount of time $T^{(b)}$ using the normal derivative of the Green's function $g(z, b)$. If we start over and do this in the other order (but for the same amount of time at each sink), we will acheive the same final domain at time $T^{(a)}+T^{(b)}$. In other words, each sink generates a flow on the space of domains containing it, and the flows generated by different sinks commute. For a proof, see [24, Section 2.4]. The fact that the flows commute is surprising, and it is not the case for lemniscate growth.

The infinitesimal version of the commuting flows can be seen as a consequence of Hadamard's variational formula. Consider a perturbation of the boundary of a domain in the normal direction, by $\delta n=\varepsilon p(s)$ where $p(s)$ is a function of arc length $s$. We recall [6, Chapter 15] that the first variation of the Green's function is

$$
\delta g_{\Omega}(z, b)=-\int_{\partial \Omega} \partial_{n} g_{\Omega}(\zeta, b) \partial_{n} g_{\Omega}(\zeta, z) \delta n d s(\zeta)
$$

Let $T^{(a)}$ and $T^{(b)}$ denote the time parameters with respect to the two flows generated by Laplacian growth with singularity at $a$ and respectively $b$. Consider the perturbation of $\Omega_{0}$ by the Laplacian growth with singularity at a. So, $p(s)=\partial_{n} g_{\Omega_{0}}(\zeta, a)$ :

$$
\frac{\partial g_{\Omega_{0}}(z, b)}{\partial T^{(a)}}=-\int_{\partial \Omega} \partial_{n} g_{\Omega_{0}}(\zeta, b) \partial_{n} g_{\Omega_{0}}(\zeta, z) \partial_{n} g_{\Omega_{0}}(\zeta, a) d s(\zeta)
$$

By virtue of the symmetry in $a$ and $b$ of the right-hand-side above, we have

$$
\frac{\partial g_{\Omega_{0}}(z, b)}{\partial T^{(a)}}=\frac{\partial g_{\Omega_{0}}(z, a)}{\partial T^{(b)}} .
$$

From this equation one can see that taking infinitesimal steps, the two flows commute. This is often described as a "zero-curvature" condition.

Let us compare this to the case of lemniscate growth. With $S^{(a)}$ and $S^{(b)}$ denoting the two time parameters for lemniscate growth with singularities at $a$ and $b$, we have

$$
\frac{\partial g_{\Omega_{0}}(z, b)}{\partial S^{(a)}}=-\int_{\partial \Omega} \partial_{n} g_{\Omega_{0}}(\zeta, z) \frac{\partial_{n} g_{\Omega_{0}}(\zeta, b)}{\partial_{n} g_{\Omega_{0}}(\zeta, a)} d s(\zeta)
$$

The right-hand-side is not symmetric in $a$ and $b$. Note however, that (15) implies

$$
\frac{1}{2}\left(\frac{\partial g_{\Omega_{0}}(z, b)}{\partial S^{(a)}}+\frac{\partial g_{\Omega_{0}}(z, a)}{\partial S^{(b)}}\right) \leq-\int_{\partial \Omega} \partial_{n} g_{\Omega_{0}}(\zeta, z) d s(\zeta)=-2 \pi
$$

with equality only for $a=b$, which tells us that the combined change in $g_{\Omega_{0}}$ is at least as large as in the case when the two poles $a$ and $b$ coincide. 
Thus, lemniscates growths with different poles do not commute, and this leads us to ask if there is a process related to lemniscate growth which does generate commuting flows.

For a one-parameter family of Jordan curves $\Gamma_{t}$, let $g_{t}\left(z, z_{E}\right)$ be the Green's function of the exterior of $\Gamma_{t}$ with $z_{E}$ a point in the exterior of $\Gamma_{t}$. Let $z=z_{I}$ be a point in the interior. Suppose also that $z=0$ is in the interior of $\Gamma_{t}$. Consider the growth law

$$
V_{z_{I}}(z)=\frac{\log \left|1-\frac{z_{I}}{z}\right|}{\partial_{n} g_{t}\left(z, z_{E}\right)}
$$

Let $R^{(a)}$ and $R^{(b)}$ denote the time parameters with respect to the two flows generated by two alternative choices $z_{I}=a$ and $z_{I}=b$ (but with $z_{E}$ fixed). We will prove the following commutation result again using the Hadamard variational formula.

$$
\frac{\partial V_{b}(z)}{\partial R^{(a)}}=\frac{\partial V_{a}(z)}{\partial R^{(b)}}
$$

First consider the variation of the Green's function $g\left(z, z_{E}\right)$ with respect to the flow generated by $z_{I}=a$ :

$$
\frac{\partial g\left(z, z_{E}\right)}{\partial R^{(a)}}=-\int_{\partial \Omega_{0}} \partial_{n} g\left(\zeta, z_{E}\right) \frac{\partial_{n} g(\zeta, z) \log \left|1-\frac{a}{\zeta}\right|}{\partial_{n} g\left(\zeta, z_{E}\right)} d s(\zeta) .
$$

Cancellation in the integrand above leads to a Poisson integral of a harmonic function,

$$
\int_{\partial \Omega_{0}} \partial_{n} g(\zeta, z) \log \left|1-\frac{a}{\zeta}\right| d s(\zeta)=2 \pi \log \left|1-\frac{a}{z}\right|
$$

Next we consider

$$
\frac{\partial V_{b}(z)}{\partial R^{(a)}}=-\frac{\log \left|1-\frac{b}{z}\right| \frac{\partial g\left(z, z_{E}\right)}{\partial R^{(a)}}}{\left(\partial_{n} g\left(z, z_{E}\right)\right)^{2}}=-2 \pi \frac{\log \left|1-\frac{b}{z}\right| \log \left|1-\frac{a}{z}\right|}{\left(\partial_{n} g\left(z, z_{E}\right)\right)^{2}} .
$$

We notice that this is symmetric in $a$ and $b$ so that we obtain the zerocurvature condition (17)

Note that this also works if you exchange $\log |1-a / z|$ in $V_{a}$ for any $h_{a}(z)$ where $h_{a}(z)$ is harmonic in $\Omega_{0}$. Perhaps in that generality it connects to something previously studied. 


\section{Certain lemniscates do survive certain elliptic growths}

In the first Section, we observed that a family of lemniscate domains such as

$$
\Omega_{t}=\left\{z \in \mathbb{C}|| z^{2}-1 \mid>\exp (2 t)\right\}
$$

has boundary velocity proportional to the reciprocal of harmonic measure at infinity. Starting from a value of $t>0$ and letting $t$ decrease, as $t$ passes through $t=0$ (Bernoulli's lemniscate) the curve breaks into two curves forming 90-degree angles (we note that during bubble break-up in Laplacian growth, instead of a 90-degree angle there are cusps).

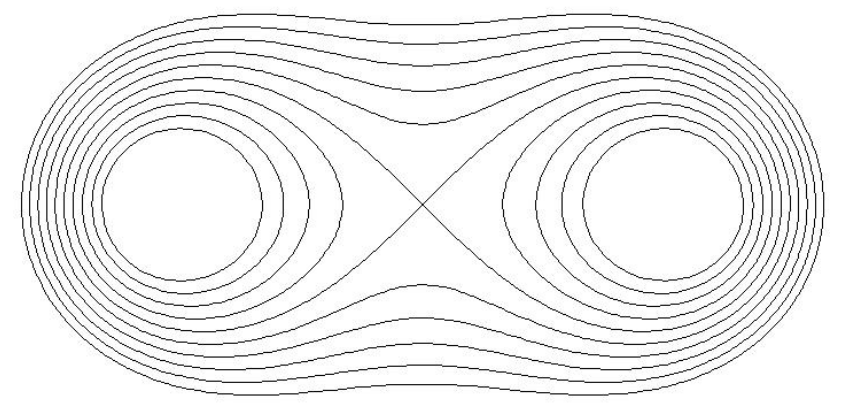

Fig. 1 Elliptic growth with $\rho=|z|^{2}$ and $\lambda=1 / \rho$ and sinks located at $z= \pm 1$.

As observed in Section 5, the complementary domains also satisfy the lemniscate growth law, but with two singularities (positioned at \pm 1 ). We will see that the same process can furthermore be viewed as a so-called "elliptic growth". Following [11], generalize the Laplacian growth process by introducing the functions $\lambda(x, y)$ and $\rho(x, y)$ as follows. Let the boundary velocity satisfy $v=\lambda \partial_{n} p$, where $p$ satisfies $\operatorname{div}(\rho \lambda \nabla p)=0$, and has prescribed singularities.

Take $\rho=|z|^{2}$, and $\lambda=\frac{1}{\rho}=\frac{1}{|z|^{2}}$. The case $\lambda=\frac{1}{\rho}$ reduces to a particularly simple instance of elliptic growth which has been called "weighted Laplacian growth". The particular choice $\rho=|z|^{2}$ (and $\lambda=\frac{1}{|z|^{2}}$ ), can be interpreted as limiting equilibrium shapes of an electron cloud confined by a quartic external field [10].

To see that (20) is an elliptic growth with the specified values of $\rho$ and $\lambda$, we rely on the "elliptic Schwarz potential" and the criterion given by $[16$, Theorem 2.2].

First recall that the Schwarz function of a real-analytic curve is the unique, complex-analytic function that coincides with $\bar{z}$ on the curve [4]. An evolution of domains is a Laplacian growth if the singularities of the Schwarz function $S(z, t)$ of the domain are independent of time except for simple poles located at the sinks whose residues change with the rates $\nu_{i}$ appearing in (12). 
This can be generalized to higher dimensions and to the case of elliptic growth by defining an elliptic Schwarz potential. It is defined to be the solution of the following Cauchy problem.

$$
\left\{\begin{array}{l}
\operatorname{div}(\lambda \rho \nabla u)=0 \text { near } \Gamma \\
\left.u\right|_{\Gamma}=q \\
\left.\nabla u\right|_{\Gamma}=\nabla q
\end{array},\right.
$$

where the function $q$ is required to satisfy the Poisson equation $\Delta q=\rho$.

In the case we are considering $\rho=|z|^{2}$, and so we take $q=\frac{|z|^{4}}{12}$. Since $\lambda=1 / \rho$, the equation $\operatorname{div}(\lambda \rho \nabla u)=0$ is just $\Delta u=0$. Since $u$ is harmonic, $u_{z}=\frac{1}{2}\left(u_{x}-i u_{y}\right)$ is analytic. Also, the Cauchy data in (21) gives us $u_{z}=$ $\frac{1}{12} z \bar{z}^{2}$ on $\Gamma$. We can replace $\bar{z}$ by $S(z)$, the Schwarz function of $\Gamma$. Moreover, since both sides of the equation

$$
u_{z}=\frac{1}{12} z S(z)^{2}
$$

are complex analytic, the equation is valid not only on $\Gamma$ but everywhere the right-hand-side is defined.

The Schwarz function for the domain described by $(20)$ is $S(z)=\sqrt{1+\frac{a}{z^{2}-1}}$, $a=\exp (2 t)$. So we have

$$
u_{z}=\frac{1}{12} z\left(1+\frac{a}{z^{2}-1}\right) .
$$

Thus, even though the (complex analytic) Schwarz function has a branch cut, the elliptic Schwarz potential has only logarithmic singularities $\left(u_{z}\right.$ has simple poles at $z= \pm 1$ with residue determined by $a$ ). This is exactly what is required according to [16, Theorem 2.2] in order for the family of domains to be an elliptic growth with "sinks" at $z= \pm 1$.

\section{Remarks}

1. In the above, we relied on electrostatics to provide a physical interpretation. However, to further pursue the comparison to Laplacian growth, we can describe a loose connection to non-Newtonian fluids.

In a Hele-Shaw cell, for an interface between an inviscid fluid and a viscous, non-Newtonian fluid, viscosity is allowed to depend on the gradient of pressure. Then the Darcy law states that the boundary velocity $v$ is the gradient of the pressure divided by a function of the gradient of the pressure. Thus, $v=$ $\frac{\nabla p}{\mu\left(|\nabla p|^{2}\right)}$, where the viscosity $\mu$ depends on $|\nabla p|^{2}$. If $\mu$ is monotone increasing, the fluid is called "shear-thickening" (e.g., corn-starch and water). If it is monotone decreasing, the fluid is called "shear-thinning" (e.g., ketchup). In the case of (2), $\mu\left(|s|^{2}\right)=|s|^{2}$, so it is shear-thickening, and this choice of $\mu$ seems like a simple one to consider. In fluid dynamics literature [5], a more 
common choice for the viscosity is $\mu\left(|s|^{2}\right)=\frac{1+\alpha|s|^{2}}{1+|s|^{2}}$, but some papers [15] do consider a power-law viscosity like our case.

Unfortunately, in non-Newtonian Hele-Shaw flows, imposing a divergencefree condition means that instead of being harmonic, the pressure now satisfies the nonlinear equation $\operatorname{div}\left(\frac{\nabla p}{\mu\left(|\nabla p|^{2}\right)}\right)=0$. In some studies [2], though, the pressure was still assumed harmonic in order to test separately the effects of changing the PDE versus the effect of changing the Darcy's law at the boundary. In this way, lemniscate growth might be viewed as a simplified model of a special case of a non-Newtonian Hele-Shaw flow involving a fluid that is shear-thickening.

2. Let $\left\{\mathcal{L}_{t}\right\}$ be a Laplacian growth process with parameter $\nu$ and with pole at $\infty$, and let $L_{t}$ be the boundary curves. Is it possible that for infinitely many $t$ the $L_{t}$ are the same as lemniscates of a Green's function with pole at $\infty$, i.e. can there be $t_{n} \rightarrow t_{0}$ and $\theta_{n} \rightarrow \theta_{0}$ such that $\Omega_{t_{n}}=\mathcal{L}_{\theta_{n}}$ where $\Omega_{t}$ is a lemniscate growth process with pole at $\infty$ and with parameter $\lambda$ ? By the computation made in the beginning, the distance from a point $z \in \Gamma_{t_{0}}$ to $\Gamma_{t_{n}}$ is about $\left(t_{n}-t_{0}\right) \lambda / D_{\mathbf{n}}(z)$ where $D_{\mathbf{n}}(z)$ denotes the normal derivative, while in Laplacian growth the distance from $z \in L_{\theta_{0}}$ to $L_{\theta_{n}}$ is about $\left(\theta_{n}-\theta_{0}\right) \nu D_{\mathbf{n}}(z)$. Thus, if $\Gamma_{t_{n}}=L_{\theta_{n}}$, then $D_{\mathbf{n}}(z)=$ const on $\Gamma_{t_{0}}$. In the simply connected case if $\varphi^{-1}$ is the conformal map from $\Omega_{t_{0}}$ onto the exterior of the unit disk, then $D_{\mathbf{n}}(z)=\left|\left(\varphi^{-1}\right)^{\prime}(z)\right|$, so we have $\left|\left(\varphi^{-1}\right)^{\prime}(z)\right|=$ const, which implies $\left|\varphi^{\prime}(w)\right|=$ const on the unit circle. But $\log \left|\varphi^{\prime}(w)\right|$ is a harmonic function outside the unit disk (also at $\infty$ ), hence it follows that it is constant, since it is constant on the boundary. Thus, $\varphi(z)=c z+d$, and $\Gamma_{t_{0}}=L_{\tau_{0}}$ is a circle (in which case then all $\Gamma_{t}, L_{t}$ are circles). Note that this is an alternative version of the theorem mentioned in the Introduction to the effect that lemniscates are not preserved under Laplacian growth.

3. In the higher-dimensional case, the condition (2) has appeared in a more general setting relating to motion of hypersurfaces in Brane Theory. The lemniscate growths we have considered give exact solutions to the twodimensional case if we assume in [3, Eq. (17) on p. 6] that $\tau$ is harmonic, which was referred to as a "time-harmonic flow".

It seems that the natural analogue of lemniscates in higher dimensions are level sets of a function which is a superposition of fundamental solutions to the Laplace equation, i.e., level sets of:

$$
f(\mathbf{x})=\sum_{i=1}^{m} \frac{c_{i}}{\left|\mathbf{x}-\mathbf{x}_{i}\right|^{n-2}},
$$

where $c_{i}$ are constants, and $\mathbf{x}_{i} \in \mathbb{R}^{n}$, or more generally, level sets of potentials

$$
f(\mathbf{x})=\int \frac{1}{|\mathbf{x}-\xi|^{n-2}} d \mu(\xi)
$$

4. Part of this paper has been a comparison of quadrature domains and lemniscates. We note that each can be used to approximate any domain in the plane having compact boundary. Approximation by lemniscates is established 
in Hilbert's lemniscate Theorem [19], and for approximation by quadrature domains, see [7] (cf. [1]).

It would be nice to find some natural interpolation between lemniscates and quadrature domains. For instance, given $n$ point masses find a smooth one-parameter family of real-analytic domains $\left\{\Omega_{t}\right\}_{t \in[0,1]}$, so that $\Omega_{0}$ is the quadrature domain whose exterior potential coincides with that of the $n$ point masses, and $\Omega_{1}$ is the lemniscate domain whose equilibrium measure generates the same exterior potential as the $n$ point masses. Can the homotopy be carried out in a way that each intermediate domain also has some potential-theoretic equivalence to the same $n$ point masses?

Acknowledgement: We wish to thank Björn Gustafsson, Jens Hoppe, Dmitry Khavinson, Ed Saff, and Razvan Teodorescu for discussions and helpful suggestions.

\section{References}

1. S. Bell, Density of quadrature domains in one and several complex variables, Complex Variables and Elliptic Equations, 54 (2009), 165 - 171.

2. D. Bonn, H. Kellay, M. Ben Amar, J. Meunier, Viscous finger widening with surfactants and polymers, Phys. Rev. Let. 75 (1995), 2132-2135.

3. M. Bordemann, J. Hoppe, Diffeomorphism invariant integrable field theories and hypersurface motions in Riemannian manifolds, arXiv:hep-th/9512001

4. P. J. Davis The Schwarz Function and its Applications, The Mathematical Association of America, Buffalo, N. Y., 1974. The Carus Mathematical Monographs, No. 17.

5. P. Fast, L. Kondic, M. J. Shelley, P. Palffy-Muhoray, Pattern formation in nonNewtonian Hele-Shaw flow, Physics of Fluids, 13 (2001), 1191-1212.

6. P. Garabedian, Partial Differential Equations, 2nd ed., Chelsea Pub. Co., 1998.

7. B. Gustafsson, Quadrature identities and the Schottky double, Acta Applicandae Math. 1 (1983), 209-240.

8. B. Gustafsson, M. Putinar, E. B. Saff, N. Stylianopoulos, Bergman polynomials on an archipelago: estimates, zeros and shape reconstruction, Adv. Math. 222 (2009), 14051460.

9. B. Gustafsson, M. Sakai, On potential theoretic skeletons of polyhedra, Geom. Dedicata, 76 (1999), 1-30

10. H. Hedenmalm, N. Makarov, Coulomb gas ensembles and Laplacian growth, preprint (2011): arxiv.org/abs/1106.2971

11. D. Khavinson, M. Mineev-Weinstein, M. Putinar, Planar elliptic growth, Complex Analysis and Operator Theory, 3 (2009), 425 - 451.

12. D. Khavinson, M. Mineev-Weinstein, M. Putinar, and R. Teodorescu, Lemniscates do not survive Laplacian growth, Mathematical Research Letters, 17 (2010), 335 - 341.

13. I. Krichever, M. Mineev-Weinstein, P. Wiegmann, A. Zabrodin, Laplacian growth and the Whitham equations of soliton theory, Physica D. 198 (2004), 1-28.

14. N. S. Landkof, Foundations of Modern Potential Theory, Grundlehren der mathematischen Wissenschaften, 180, Springer-Verlag, Berlin, New York, 1972

15. A. Lindner, D. Bonn, and J. Meunier, Viscous fingering in a shear-thinning fluid, Phys. Fluids 12, 256 (2000).

16. E. Lundberg, Laplacian growth, elliptic growth, and singularities of the Schwarz potential, J. Phys. A: Math. Theor., 44 (2011), 135202.

17. E. Miña-Diaz, E. B. Saff, N. Stylianopoulis, Zero distributions for polynomials orthogonal with weights over certain planar regions, Computational Methods and Function Theory, 5 (2005), $185-221$.

18. C. Pommerenke, Univalent Functions, Vandenhoeck und Ruprecht, Gottingen, 1975. 
19. T. Ransford, Potential Theory in the Complex plane, Cambridge University Press, Cambridge, 1995.

20. S. Richardson, Hele-Shaw flows with a free boundary produced by the injection of fluid into a narrow channel, J. Fluid Mech., 56 (1972), 609 - 618.

21. E. B. Saff, private communication

22. E. B. Saff and V. Totik, Logarithmic Potentials with External Fields, Grundlehren der mathematischen Wissenschaften, 316, Springer Verlag, Berlin, Heidelberg, 1997.

23. M. Tsuji, Potential Theory in Modern Function Theory, Maruzen, Tokyo, 1959.

24. A. N. Varchenko, P. I. Etingof, Why the boundary of a round drop becomes a curve of order four, AMS university lecture series Vol. 3, 1992. 